Bangladesh J. Plant Taxon. 21(2): 105-120, 2014 (December)

(C) 2014 Bangladesh Association of Plant Taxonomists

\title{
REVISION OF AMORPHOPHALLUS BLUME EX DECNE. SECT. AMORPHOPHALLUS (ARACEAE) IN INDIA
}

\author{
V. Abdul Jaleel ${ }^{1}$, M. Sivadasan ${ }^{2,3}$, Ahmed H. Alfarhan ${ }^{2}$, \\ Jacob Thomas ${ }^{2}$ and A. A. Alatar ${ }^{2}$ \\ Department of Botany, University of Calicut, Calicut University P. O., 673 635, Kerala, India
}

Keywords: Amorphophallus sect. Amorphophallus; Araceae; Endemics; India.

\begin{abstract}
Amorphophallus Blume ex Decne. sect. Amorphophallus in India is revised. It is the smallest of the three sections in India with five species, viz. A. hirsutus Teysm. \& Binn., A. kachinensis Engl. \& Gehrm., A. longistylus Kurz, A. napalensis (Wall.) Bogner \& Mayo and A. paeoniifolius (Dennst.) Nicolson. Amorphophallus paeoniifolius is the widely distributed species in India with two varieties, viz. A. paeoniifolius var. paeoniifolius, and var. campanulatus (Decne.) Sivad. Amorphophallus longistylus is the only species of the section endemic to India.
\end{abstract}

\section{Introduction}

Amorphophallus Blume ex Decne. sect. Amorphophallus (Araceae) is the smallest of the three sections represented in India. Revisions of the other two sections, viz. Rhaphiophallus and Conophallus have been carried out recently by Jaleel et al. (2011, 2012).

Engler (1911) in his revision of the genus treated Amorphophallus campanulatus Decne. [=A. paeoniifolius (Dennst.) Nicolson] under the section Cundarum Engl. which is a renaming of Candarum Rchb. ex Schott. According to Engler (1911), composition of the name 'Candarum' was improper and he renamed it as 'Cundarum' stating that the name is derived from the Indian name 'Kunda' (= Amorphophallus campanulatus) to which the suffix 'arum' was added resulting in 'Kundarum' and written as 'Cundarum'. He considered it as a new name with his authorship. But as per Art. 60.1 of ICN (McNeill et al., 2012) the original spelling of a name or epithet is to be retained, and hence the name Cundarum Engl. is treated as illegitimate. Engler (1911) included Amorphophallus campanulatus, the type of the genus Amorphophallus under the section Cundarum Engl. As per Art. 22.1. of ICN, the name of any subdivision of a genus that includes the type of an adopted, legitimate name of the genus to which it is assigned is to repeat that generic name unaltered as its epithet, and accordingly the correct name of the section of Amorphophallus that includes Amorphophallus campanulatus [=A. paeoniifolius] should have been Amorphophallus sect. Amorphophallus, and it is used in the present study.

Out of the seventeen species of Amorphophallus included in Flora of British India by Hooker (1894), only eight were reported to be occurring in India and Amorphophallus longistylus Kurz ex Hook. f., A. campanulatus (=A. penoniifolius) and A. dubius Blume (=A. penoniifolius) are among those belonging to the section Amorphophallus as per the present standards adopted for species delimitation. Engler (1911) in his monographic work included A. longistylus under the section Conophallus, and $A$. napalensis was treated under the genus Thomsonia which was later transferred to Amorphophallus by Bogner et al. (1985). Since Engler's work, several new species have been discovered from various parts of India. Brief accounts on earlier taxonomic work on Indian Amorphophallus have been provided by Jaleel et al. (2011, 2012). The present article is the third and final part of revision of the genus Amorphophallus in India.

\footnotetext{
${ }^{1}$ Present address: Department of Botany, Sir Syed College, Taliparamba, Kannur-670 142, Kerala, India ${ }^{2}$ Department of Botany \& Microbiology, College of Science, King Saud University, P. O. Box 2455, Riyadh11451, Kingdom of Saudi Arabia

${ }^{3}$ Corresponding author: Email: drmsivadasan@rediffmail.com
} 
The methodology adopted in earlier works (Jaleel et al., 2011, 2012) have been followed in the present work. Extensive and exhaustive field explorations covering all seasons were made all over India for collection and recording relevant data of the specimens.

Indian specimens available at various major herbaria such as ASSAM, BM, BSA, BSD, BSHL, BSI, CAL, CALI, DD, GH, JCB, K, KFRI, L, M, MH, PBL, TBGT and US were consulted, and representative specimens were cited.

\section{Taxonomic treatment}

Amorphophallus Blume ex Decne., Nouv. Ann. Mus. Hist. Nat. 3: 366 (1834), nom. cons. [Taxon 31: 310 (1982)].

Type: Amorphophallus campanulatus Decne. [= A. paeoniifolius (Dennst.) Nicolson].

Amorphophallus Blume ex Decne. sect. Amorphophallus, emend. Sivad. mut. char. [Amorphophallus sect. Candarum Blume, Rumphia 1:139 (1835). Amorphophallus sect. Cundarum Engl., Pflanzenr. IV. 23C (48): 74 (1911), nom. illegit.].

Spathe usually campanulate with a basal convolute tube and an upper horizontally spreading limb or with basal convolute tube and erect, oblong or oblong-ovate or ovate limb; appendix usually conoid, hemispheric or cylindric; style always long, equal to or 2-4 times the height of ovary; stigma lobed.

\section{Key to the Indian species of Amorphophallus sect. Amorphophallus}

1. Peduncle 3-8 cm long; spathe broadly campanulate with basal convolute tube and horizontally spreading limb; spadix-appendix sub-globose or conoid.

- Peduncle more than $20 \mathrm{~cm}$ long; spathe ovate with basal convolute tube and upper erect open limb; spadix-appendix elongate-ovoid or cylindric.

2. Spadix-appendix with subglobose base and apical short cylindric truncate column bearing short stiff hairs.

A. hirsutus

- Spadix-appendix subglobose or conoid, glabrous.

A. paeoniifolius

3. Petiole and peduncle smooth with even surface; spadix shorter than spathe; appendix elongate-ovoid with irregular longitudinal grooves or fissures, or cylindric with warts; style length more or less equal to the height of ovary.

- Petiole and peduncle smooth with uneven surface having small bumps; spadix longer than spathe; appendix elongate, cylindric with tapered tip, smooth; style 2-3 times the height of ovary.

4. Spadix stipitate; appendix elongate-ovoid, surface with irregular longitudinal grooves or fissures; style straight; stigma inconspicuously 3-lobed.

A. kachinensis

- Spadix sessile; appendix cylindric, surface rough with short prominent protuberances; style bent towards spadix-axis; stigma 4-lobed.

A. napalensis

Amorphophallus hirsutus Teysm. \& Binn., Naturk. Tijdschr. Nederl. Ind. XXIV: 332 (1862); Engl., Pflanzenr. IV. 23C (48): 106 (1911); Sivad. \& Jaleel, Rheedea 10(2): 143 (2000) (Fig. 1).

Type: West Sumatra, Soeka Menanti, Ophir, (no date), Buennemeijer 1019 (Neotype: BO).

Tubers depressed-globose, 4.5-8.5 cm diam. and 2.5-5.0 cm thick in vegetative phase; c. 11 $\mathrm{cm}$ diam. and $7 \mathrm{~cm}$ thick in reproductive phase; root scars thickened, annulate. Petiole 73-82 cm 
long, pale green with small blackish green irregular specks and mottles with minute dark green spots in between, paler towards the tip, extreme base with purplish blue hue; some petioles with pale green background having large irregular blackish green patches with light greyish margin, and minute greenish spots in between, and paler towards the tip. Leaflets sessile, ellipticlanceolate, large leaflets 11-17 × 3-5 cm, small 6.2-10.5 × 1.8-4.0 cm, acuminate at apex, base unequal, decurrent on rachis, greenish above and pale below. Peduncle short, 3.0-5.5 cm long, greenish in colour. Spathe campanulate, broadly triangular-ovate, c. $17.5 \times 26.0 \mathrm{~cm}$, basal tube separated from limb by a constriction, tip acute, margin undulate; tube c. $7.5 \mathrm{~cm}$ diam. and $5.5 \mathrm{~cm}$

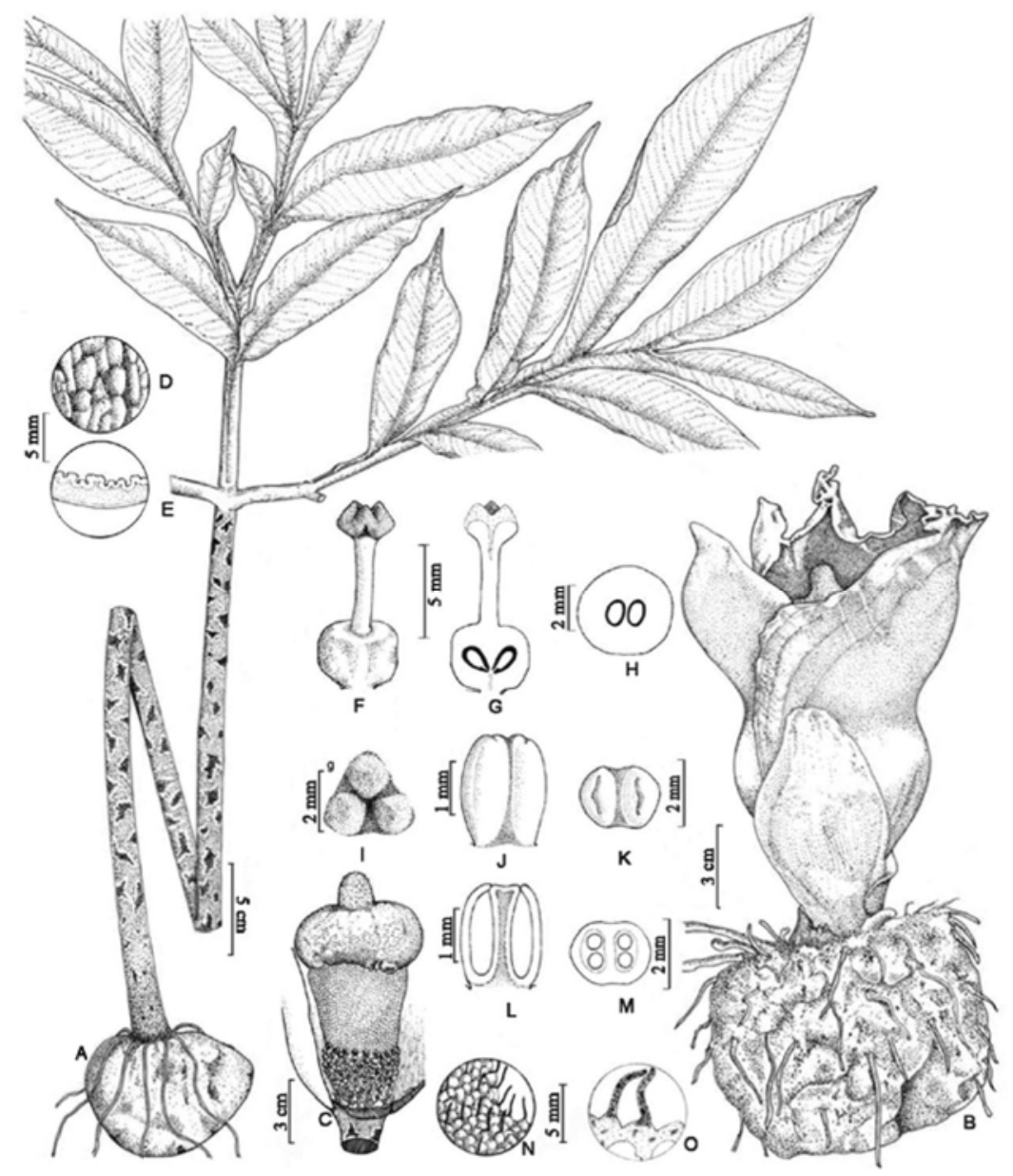

Fig. 1. Amorphophallus hirsutus Teysm. \& Binn. A. Tuber with leaf; B. Tuber with inflorescence; C. Inflorescence - spathe partially removed showing spadix; D. Small basal inside portion of spathe; E. A small basal portion of spathe - c.s.; F. Female flower; G. Female flower - l.s.; H. Ovary - c.s.; I. Stigma - view from top; J. Male flower - view from broad side; K. Male flower - view from top; L. Male flower - l.s.; M. Male flower - c.s.; N. A small portion of apical portion of spadix-appendix showing papillae; O. Appendix papillae - enlarged. 
high, greenish outside with few small white mottling, smooth, pale greenish inside, purplishorange or maroon at extreme base, rough, irregularly and longitudinally rugose and verrucate; limb c. $9.5 \mathrm{~cm}$ long spreading, up to c. $12 \mathrm{~cm}$ diam., purplish outside and inside. Spadix shorter than spathe, c. $12.5 \mathrm{~cm}$ long; sessile, female zone c. $3.3 \mathrm{~cm}$ long; male zone c. $3.8 \mathrm{~cm}$ long; appendix c. $3 \mathrm{~cm}$ high and c. $5.5 \mathrm{~cm}$ diam. Female flowers dense, each flower c. $10 \mathrm{~mm}$ high; ovary subglobose, pale greenish, c. $4 \mathrm{~mm}$ diam. and $3 \mathrm{~mm}$ high, 2-3-locular, each locule with a single basal anatropous ovule; style c. $5 \mathrm{~mm}$ long, purplish; stigma 2-3-lobed, c. $2.5 \mathrm{~mm}$ diam., pale yellowish. Male flowers dense, pale yellowish with purplish tinge at top of connectives; each c. $2 \mathrm{~mm}$ high, sessile, inconspicuously 2-lobed. Spadix-appendix subglobose, light purplish yellow, rough, abruptly narrowed to a cylindric truncate column of c. $1.8 \mathrm{~cm}$ high and $0.9 \mathrm{~cm}$ diam., light purplish yellow, rough; cylindric column and its basal surrounding portion covered with stiff slender bulbous-based papillae; papillae on cylindrical column smaller compared to that of basal neighbouring portion; appendix becomes unevenly bullate after anthesis.

Phenology: Flowering: May; fruiting: Fruiting specimens could not be collected.

Representative specimens examined: Andaman and Nicobar Islands: Great Nicobar Island, On the way to East-West Road, 17.5.1999, Abdul Jaleel RIA 350 (Infl.) (CALI); Ibid., 17.5.1999, Abdul Jaleel RIA 351 (tuber and leaf) (CALI); Ibid., 20.5.1999, Abdul Jaleel RIA 355 (tuber and leaf) (CALI). Kerala: Calicut University Botanical Garden, 17.4.2000, Abdul Jaleel RIA 382 (Infl.) (CALI) (originally collected from East West Road, Great Nicobar Island, and introduced and flowered in the Calicut University Botanical Garden).

Notes: Amorphophallus hirsutus resembles A. paeoniifolius and A. prainii in general vegetative morphology and inflorescence, especially during the early stages. It differs from the latter two by having a subglobose appendix with a cylindric apical column covered with short stiff papillae. There is no other Indian species with hairs on the spadix-appendix.

Distribution: Originally collected from Java and Hetterscheid and Ittenbach (1996) reported its occurrence in Western Sumatra. Sivadasan and Jaleel (2000) first reported it from Great Nicobar islands of India where it is rare.

Amorphophallus kachinensis Engl. \& Gehrm. in Engler, Pflanzenr. IV. 23C (48): 91 (1911); Hett. \& Itten., Aroideana 19: 87 (1996).

(Fig. 2).

Type: Upper Burma, Kachin Hills, 20.5.1898, Shaik Mokim, s.n. (Holotype: CAL).

Tubers depressed-globose, 5-30 cm diam. and 3-5 cm thick, skin brownish; produce offsets. Petiole c. $20 \mathrm{~cm}$ long, smooth, dirty white background with green to reddish brown spots. Leaflets elliptic, 6-9 $\times 2-3 \mathrm{~cm}$, tip acute-acuminate. Peduncle $24-80 \mathrm{~cm}$ long. Spathe more or less boatshaped, slightly convolute at base, $8-29 \times 7-14 \mathrm{~cm}$, tip rounded or truncate, green or greenish brown outside with green spots or purplish stripes and spots; light purplish within, with scattered, shallow, punctiform warts at base. Spadix much shorter than spathe, 6.5-18.0 cm long, stipitate, stipe 0.2-1.0 cm long; female zone c. $5 \mathrm{~cm}$ long; male zone c. $7.5 \mathrm{~cm}$ long; spadix-appendix c. 15 $\mathrm{cm}$ high. Female flowers dense, each c. $2.5 \mathrm{~mm}$ high; ovary sub-globose, c. $1.5 \mathrm{~mm}$ high, unilocular with basal anatropous ovule; style c. $0.7 \mathrm{~mm}$ long; stigma inconspicuously 3-lobed. Male flowers dense, each c. $2 \mathrm{~mm}$ broad, sessile, inconspicuously 2-lobed. Spadix-appendix ellipsoid or ovoid with several irregular longitudinal grooves or fissures.

Phenology: Flowering: April - May.

Representative specimens examined: Arunachal Pradesh: Ziro, 10.4.2006, Abdul Jaleel RIA 424 (infl.) (CALI); Ibid., 7.8.2006, Abdul Jaleel RIA 430 (leaf) (CALI). 


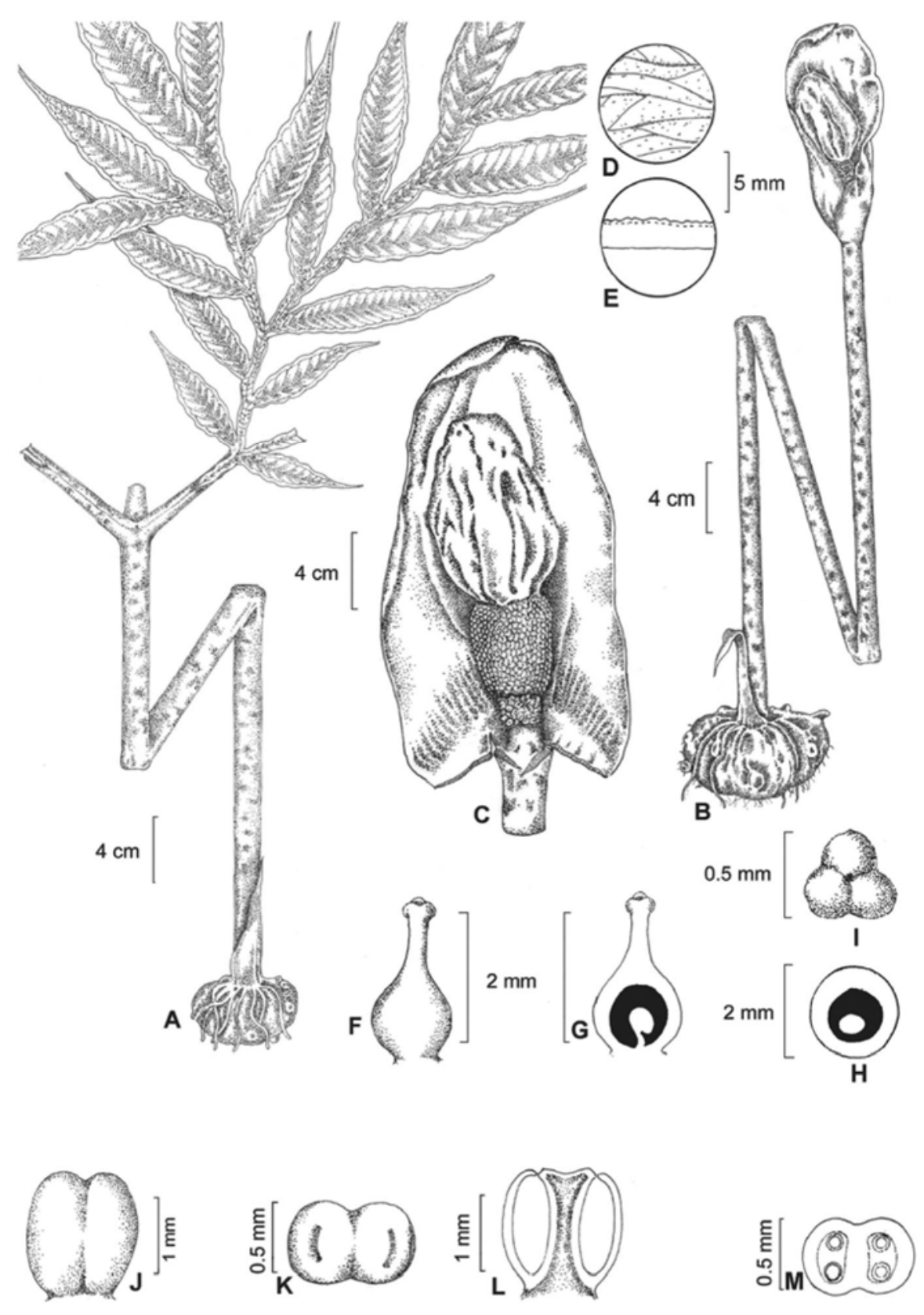

Fig. 2. Amorphophallus kachinensis Engl. \& Gehrm. A. Tuber with leaf; B. Tuber with inflorescence; C. Inflorescence - spathe cut-opened showing spadix; D. A small basal portion of spathe; E. A small basal inside portion of spathe - c.s.; F. Female flower; G. Female flower - l.s.; H. Ovary - c.s.; I. Stigma - view from top; J. Male flower - view from broad side; K. Male flower - view from top; L. Male flower - l.s.; M. Male flower - c.s. 
Notes: Amorphophallus kachinensis resembles A. corrugatus N. E. Br. and A. yunnanensis Engl. (1911) in general morphology but differs in the nature of spadix-appendix by having longitudinal irregular grooves. It also differs from A. yunnanensis in having female flowers with long style and smaller stigma.

Distribution: Northern Myanmar (Kachin state), Northern Thailand, Laos, China (Yunnan) and India. In India, it was collected from Arunachal Pradesh and present collection of the species forms a new distributional record for India.

Amorphophallus longistylus Kurz (Rep. Andaman 50.1866, nomen) ex Hook. f., Fl. Brit. India 6: 515 (1893); Engl., Pflanzenr. IV. 23 (48): 83 (1911); Sivad. \& Jaleel, Rheedea 8(1): 103 (1998).

(Fig. 3).

Types: India, South Andaman, (no date), Kurz s.n. (Holotype: CAL; Isotype: K).

Tubers sub-globose, 5.0-6.5 cm diam. and 4-5 cm thick in vegetative phase, c. $6.2 \mathrm{~cm}$ diam. and $4.2 \mathrm{~cm}$ thick in reproductive phase. Petiole $43-71 \mathrm{~cm}$ long, surface uneven with white elongated or round thickened swellings or projections; pale greenish with greenish black and white mottles. Leaflets sessile, ovate to oblong, apex acuminate, $6.5-12.0 \times 2.8-4.6 \mathrm{~cm}$; base of leaflets on secondary rachises decurrent, leaflets on primary rachises usually not decurrent-based; greenish above, paler below with pinkish tinge along the veins, margins and tip; margin undulate. Peduncle 30.5-42.5 cm long, 1.0-1.2 cm diam. Spathe erect, ovate-lanceolate, $18.5-23.5 \times 9.5-10.5 \mathrm{~cm}$, tip acute, basal convolute tube 7.5-14.0 cm high and 3.5-4.0 cm diam.; upper expanded limb portion with longitudinal shallow folds on either side of mid-portion; light purplish with greenish black blotches and small dark spots on outside, more on basal tube; smooth, dark purplish and verrucose at base, pale purplish and smooth above with few light greenish-black blotches above within. Spadix longer than spathe, exserted, 24-26 cm long, stipitate; stipe short, $0.2-0.4 \mathrm{~cm}$ long; female zone 2.4-3.2 cm; male zone 3.2-3.7 cm long; appendix 17-20 cm long. Female flowers 60-80, loosely arranged, each 5-8 mm high, ovary 1.7-2.0 mm high, shallowly 3-5-lobed, greenish yellow, 3-5-locular, each locule with a single basal anatropous ovule; style 3-7 mm long, rarely with longitudinal ridges at upper portion, dark purplish; stigma 3-5-lobed, rarely 2-lobed, creamy. Male flowers dense, each c. 2 mm high, sessile, inconspicuously 2-lobed, yellowish-creamy with purplish tinge on connective at top. Spadix-appendix cylindric, gradually tapering to tip.

Phenology: Flowering: May - June; fruiting: Fruits could not be collected.

Representative specimens examined: Andaman and Nicobar Islands: South Andaman, date nil, S. Kurz s.n. (CAL, K); Middle Andaman, Panchawati, 7.12.1997, Jaleel \& Bobby Thomas RIA 224 (leaf) (CALI); North Andaman, Ray Hill, 26.5.1999, Jaleel RIA 357 (leaf) (CALI). Kerala: Calicut University Botanic Garden, 2.5.1998, Jaleel \& Sivadasan RIA 275 (infl.) (CALI); Ibid., 21.5.1998, Jaleel \& Sivadasan RIA 227 (leaf) (CALI) (Originally collected by Jaleel and Bobby Thomas (RIA 224) on 7.12.1997 from Panchawati, Midddle Andaman and introduced in the Calicut University Botanic Garden).

Notes: This is a very rare species and was left unknown until it was re-collected in 1997 from Middle Andaman about 131 years after it was first collected by Kurz (Sivadasan and Jaleel, 1998). The species is very distinct in having uneven surface of petioles and peduncles with bumps or swellings.

Distribution: Known to occur only in Andaman Islands. 


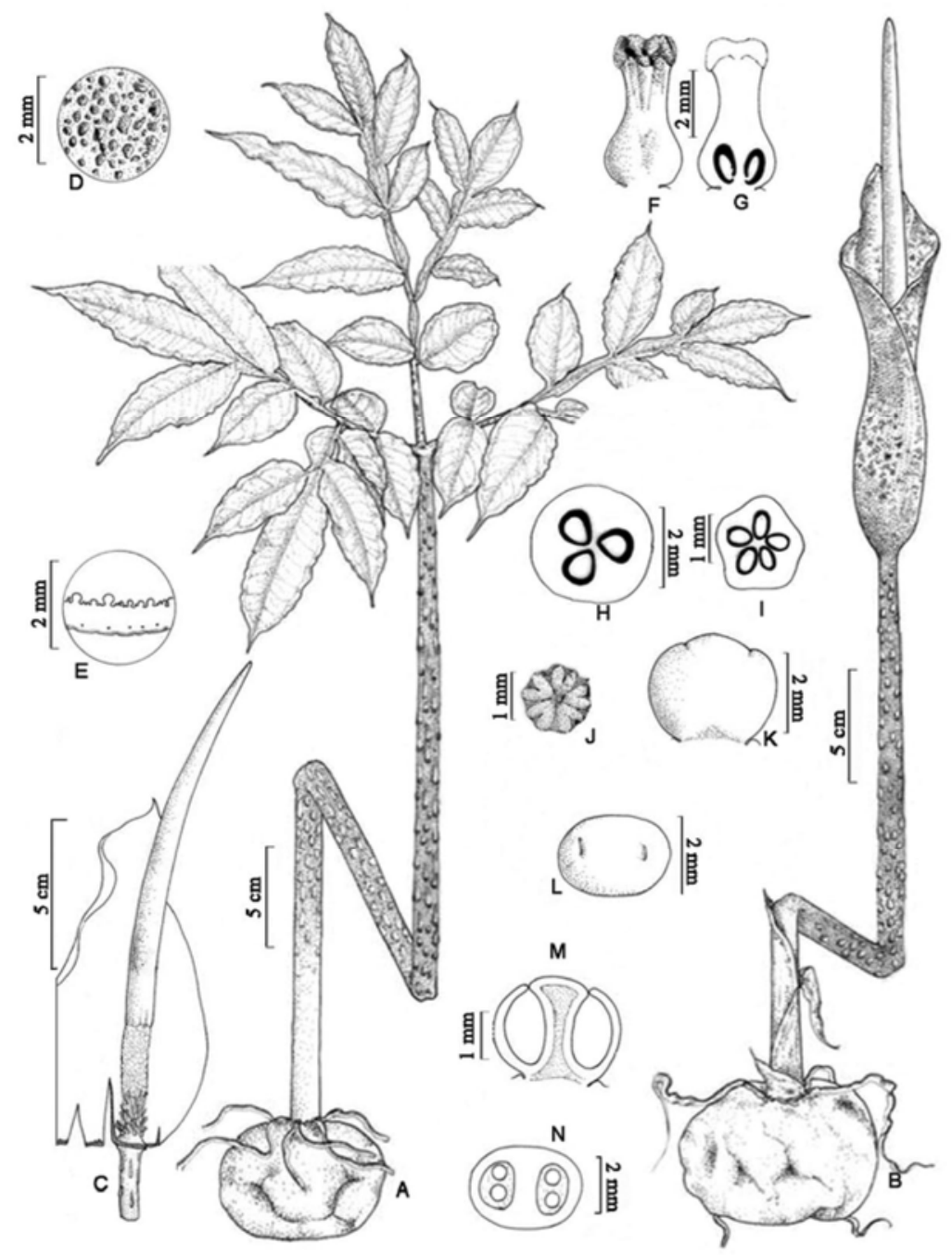

Fig. 3. Amorphophallus longistylus Kurz. A. Tuber with leaf; B. Tuber with inflorescence; C. Inflorescence spathe cut-opened showing spadix; D. Small basal inside portion of spathe; E. A small basal portion of spathe - c.s.; F. Female flower; G. Female flower - l.s.; H. Ovary with three locules - c.s.; I. Ovary with five locules - c.s.; J. Stigma of five-loculed flower - view from top; K. Male flower - view from broad side; L. Male flower - view from top; M. Male flower - l.s.; N. Male flower - c.s.

Amorphophallus napalensis (Wall.) Bogner et Mayo in Bogner et al., Aroideana 8(1): 19 (1985); Hett. \& Ittenbach, Aroideana 19: 103 (1996). Thomsonia napalensis Wall., Pl. Asiat. Rar.1: 83, t. 99 (1830); Hook. f., Fl. Brit. India 6: 518 (1893); Engl., Pflanzenr. IV. 23C (48): 56 (1911). Pythonium wallichianum Schott in Schott \& Endl., Melet. Bot. 17 (1832).

(Fig. 4).

Type: Nepal, in mountain forests, flowering in June, t. 99 (Wallich, Pl. Asiat. Rar., 1830).

Tubers subglobose or depressed-globose, $5.0-8.5 \mathrm{~cm}$ diam. and 4.5-6.5 $\mathrm{cm}$ thick in vegetative phase, 7.5-10.0 cm diam. and 5-8 $\mathrm{cm}$ thick in reproductive phase, offsets produced from tuber 
(observed only in tubers of vegetative phase), each offset $3.5-7.5 \mathrm{~cm}$ long. Petiole smooth, 41.5$78.0 \mathrm{~cm}$ long, pale green with more or less irregular or oval elongate, brown or dark brown patches. Leaflets sessile, ovate-lanceolate, large leaflets 12.2-19.2 × 5.3-6.0 cm, small leaflets 5.5$9.0 \times 2.5-4.5 \mathrm{~cm}$, marginal portion undulate, tip long-acuminate; upper surface green, lower surface pale green. Peduncle smooth, 67-76 cm long. Spathe elongate-obovate with acute tip, 24$27 \mathrm{~cm}$ long, differentiated into a basal convolute tube and open limb; tube 4.5-6.0 cm long and 5.5-7.0 cm diam., pale green with greenish brown at base outside, pale green within, changes to

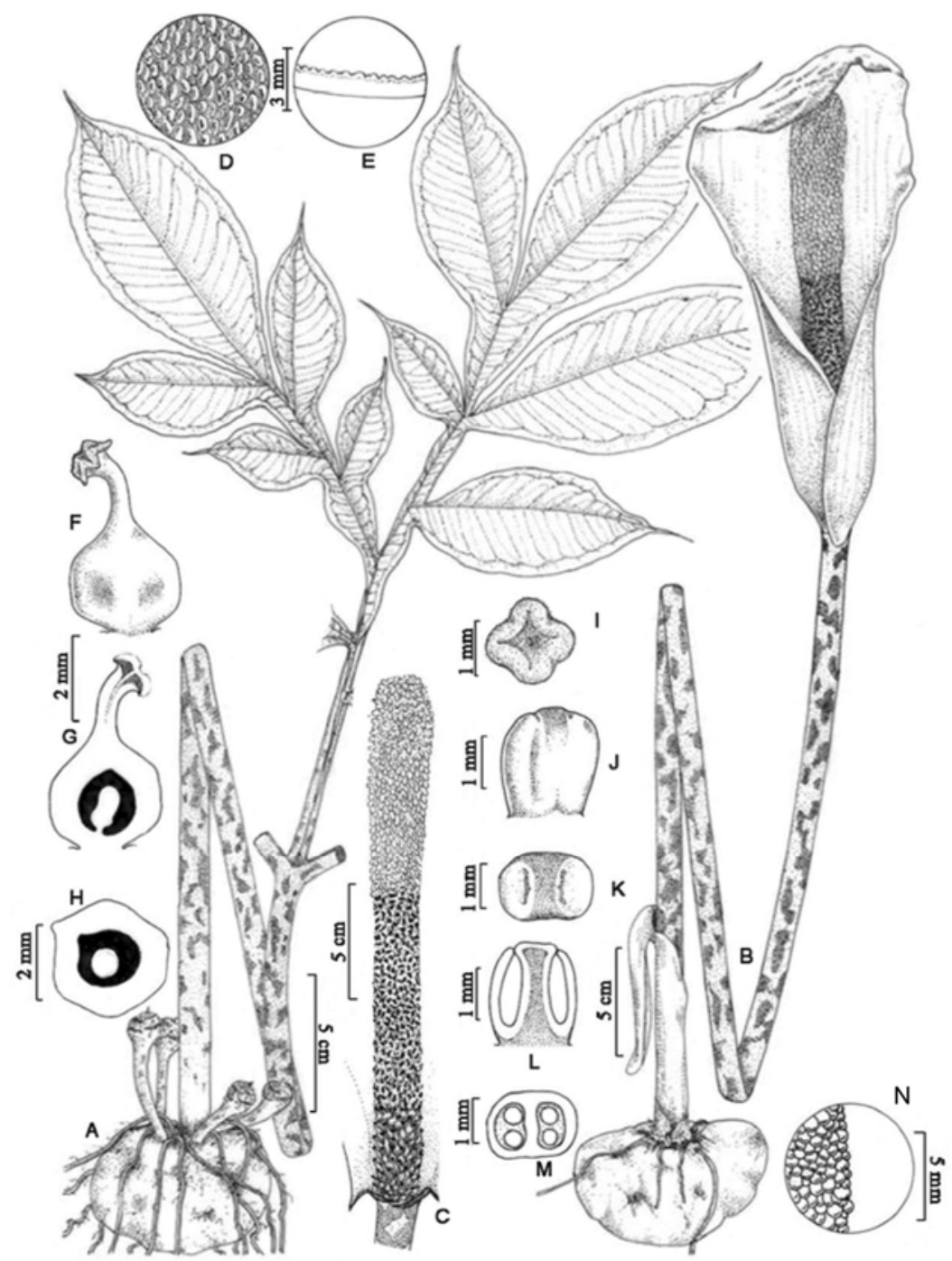

Fig. 4. Amorphophallus napalensis (Wall.) Bogner et Mayo. A. Tuber with leaf; B. Tuber with inflorescence; C. Inflorescence - spathe partially removed showing spadix; D. Small basal inside portion of spathe; E. Small basal portion of spathe - c.s.; F. Female flower; G. Female flower - l.s.; H. Ovary - c.s.; I. Stigma view from top; J. Male flower - view from broad side; K. Male flower - view from top; L. Male flower l.s.; M. Male flower - c.s.; N. A small lateral portion of spadix-appendix. 
yellowish after male anthesis, mouth of tube wide, limb expanded, become apically cucullate. Spadix sessile, shorter than the spathe, 18-20 cm long; female zone 3-4 cm long; male zone 6-7 cm long; appendix 10-12 cm long. Female flowers dense, each 4-5 mm high, ovary sub-spherical, greenish, 2.0-2.5 mm high, unilocular with single basal anatropous ovule; style c. $2 \mathrm{~mm}$ long, apically bent towards spadix-axis; stigma 4-lobed. Male flowers dense, each 2.0-2.5 mm high, inconspicuously 2-lobed, pale yellowish with reddish brown tinge on connective at top. Spadixappendix cylindric with obtuse tip, rough with prominent, short cylindric or obovoid warts, greenish when young, brownish yellow when mature, yellowish after anthesis. Fruits ovoidelliptic, 1.4-1.5 cm long. Seeds ellipsoid, 1.2-1.3 cm long.

Phenology: Flowering: May - June; fruiting: July - September.

Representative specimens examined: Sikkim: Gangtok, Deurali, 23.9.1997, Abdul Jaleel \& Bobby Thomas RIA 168 (leaf) (CALI); Ibid., 24.9.1997, Abdul Jaleel \& Bobby Thomas RIA 171 (infr.) (CALI). Assam: Assam, 29.5.1896, Prain, Acc. No. 496857 (leaf) (CAL); Chirapunji, 3.6.1956, Rolla Seshagiri Rao 2697 (infl.) (ASSAM). Meghalaya: Shillong, Woodlands, BSI Campus (Introduced; exact locality of original collection not known), 15.7.1967, Verma 35658 (infl.) (ASSAM); Shillong, Oakland, 24.6.1998, Abdul Jaleel RIA 289 (infl.) (CALI); Arunachal Pradesh: Sessa Orchid Sanctuary, 27.6.1998, Abdul Jaleel RIA 314 (leaf) (CALI).

Notes: Amorphophallus napalensis differs from other Indian species in having a verrucate appendix with short, prominent warts, style apically bent towards spadix-axis, and stigma 4-lobed.

Distribution: Bhutan, Nepal and India (Sikkim, Assam, Meghalaya and Arunachal Pradesh).

Amorphophallus paeoniifolius (Dennst.) Nicolson, Taxon 26: 338 (1977); Nicolson in Saldanha \& Nicolson, Fl. Hassan Dist. App. II : 7 (1978) ("1976"). Dracontium paeoniifolium Dennst., Schlüssel Hort. Malab. : 13, 38 (1818); Manitz, Taxon 17: 449 (1968). Arum campanulatum Roxb., [Hort. Beng. : 66 (1814), nom. nud.] Pl. Corom. 3: 68 (1819), nom. illegit. (incl. type of $D$. paeoniifolium Dennst., 1818); Wight, Icon. Pl. Ind. Or. 3: 5 (1844). Amorphophallus campanulatus Decne., Nouv. Ann. Mus. Hist. Nat. Paris 3: 336 (1834).

Lectotype: Rheede's illustration of Mulenschena in Hort. Malab. 11: t. 19 (1692), vide Nicolson, Taxon 26: 338 (1977).

Tuber depressed-globose. Petiole up to $1.2 \mathrm{~m}$ long, smooth or muricate, mottled. Inflorescence short-peduncled, elongating after anthesis. Spathe broadly campanulate, c. $25 \times 28$ $\mathrm{cm}$, convolute below and spreading above. Spadix sessile, differentiated into basal female portion, a subturbinate or subcylindric male portion and an apical naked sterile, sessile subglobose or elongate-conoid appendix, wrinkled at maturity, spongy within. Ovary light purplish or pale yellowish, 2-3-loculed, each locule with single anatropous ovule; style elongate; stigma yellowish, reniform or 2-3-lobed. Male flowers creamy-yellow.

Notes: The close morphological similarities of Schena (Hort. Malab. 11: 35, t. 18. 1692) and Mulenschena (Hort. Malab. 11: 37, t. 19. 1692) of Rheede and unawareness of the existence of an earlier legitimate epithet for Mulenschena, the name Amorphophallus campanulatus Decne. had been used for both the cultivated and wild elements represented by Schena and Mulenschena respectively. Realizing the existence of an earliest epithet for Amorphophallus campanulatus, Nicolson (1977) made a combination of name, viz. Amorphophallus paeoniifolius (Dennst.) Nicolson, as applying to Amorphophallus campanulatus (sensu lato). The wild and cultivated elements differ in many respects even though they resemble in general appearance and many other characteristics; and hence they are treated as two distinct varieties of A. paeoniifolius. Backer (1920) recognized the wild and cultivated elements as belonging to two distinct subspecific taxa and assigned the rank 'hoofdgroep' which is not valid. Backer and Bakhuizen van den Brink 
(1968) replaced the rank 'hoofdgroep' with 'forma'. Detailed accounts on the identity and nomenclature of Rheede's Schena and Mulenschena have been provided by Suresh et al. (1983).

Distribution: India, Sri Lanka and Pacific islands. In India, found in almost all States.

\section{Key to the varieties of Amorphophallus paeoniifolius}

1. Petiole usually purplish brown with light pinkish blotches, strongly muricate especially at basal half; leaflet-bases strongly decurrent on primary rachises to the main junction; spadix-appendix elongate-conoid, height more than breadth; style length about double the ovary height; stigma usually 2-lobed.

- Petiole usually greenish with white blotches, smooth, rarely slightly rough at basal half; leaflet-bases not decurrent to the junction of the primary rachises; spadix-appendix subglobose, breadth more than height; style length 3-4 times the ovary height; stigma usually 3-lobed.

var. campanulatus

Amorphophallus paeoniifolius (Dennst.) Nicolson var. paeoniifolius. Sivad. in Suresh, Sivad. \& Manilal, Taxon 32: 128 (1983); Karth., Jain, Nayar \& Sanjappa, Fl. Ind. Enum. Monocot. : 6 (1989); Sivad. in Manoharan, Biju, Nayar \& Easa, Silent Valley-Whisp. Reas.: 230 (1999). [Mulenschena Rheede, Hort. Malab. 11: 37, t. 19 (1692)]. Dracontium paeoniifolium Dennst., Schlüssel Hort. Malab.: 13, 21, 38 (1818) ('paeoniaefolium'); Manitz, Taxon 17: 499 (1968). Arum campanulatum Roxb. [Hort. Beng. 65 (1814)], Pl. Corom. 3: 68 (1820), nom. illegit.; Wight, Ic. Pl. Ind. Or. 3: 5, t. 785 (as to leaf) (1844). Candarum hookeri Schott in Schott \& Endl., Melet. Bot.: 17 (1832), nom. illegit. Kunda verrucosa Raf., Fl. Tellur. 2: 82 (1837), nom. illegit. Amorphophallus rex Prain, J. Asiat. Soc. Bengal 62: 79 (Aug. 1893); Hook. f., Fl. Brit. India 6: 514 (Sept. 1893); Engl., Pflanzenr. IV. 23C (48): 75 (1911). Amorphophallus campanulatus hoofdgroep sylvestris Backer, Determ.-tab. Jav. Amorphophallus: 2 (1920), (rankless name). Amorphophallus campanulatus f. sylvestris Backer ex Backer \& Bakh., Fl. Java 3: 112 (1968).

(Fig. 5).

Type: Same as of the species. [Lectotype: Rheede's illustration of Mulenschena in Hort. Malab. 11: t. 19 (1692), vide Nicolson, Taxon 26: 338 (1977)].

Tubers depressed-globose, 9-14 cm diam. and 8-11 cm thickness in vegetative phase; 13-20 $\mathrm{cm}$ diam. and 7-9 $\mathrm{cm}$ thickness in reproductive phase; skin pale brown to dark brown with prominent root scars. Petiole rough, 55-105 cm long, dark brownish green with round to ovoid green blotches, extreme base pinkish with minute pale greenish dots above. Leaflets sessile, large leaflets 17-20 × 5-6 cm, small leaflets 8-10 × 2.5-3.5 cm, obovate, tip acute, base unequal and decurrent on rachis, greenish above, paler below. Peduncle short, rough, 3-6 cm long. Spathe margin undulate, tip acute, 10-21 × 12-22 cm; outside greenish yellow with green blotches at base, pale brownish with pale yellow blotches above; brownish green with prominent murications at base within, pale green above. Spadix sessile, 15.5-16.0 cm long; female zone 4-5 cm long; male zone 3.5-4.5 cm long; appendix 9.5-10.5 cm long, 7-8 cm diam. at base. Female flowers dense, each with ovary c. $2 \mathrm{~mm}$ high, sub-globose, style $4-5 \mathrm{~mm}$ long, pinkish brown; stigma 2-lobed, rarely inconspicuously 3-lobed. Male flowers dense, each 4.0-4.5 cm high, cream-coloured. Spadix-appendix dark purplish red. Fruits green, reddish at maturity, each 1.6-1.8 cm long. Seeds 1-2, each 1.1-1.3 cm long.

Phenology: Flowering: May - June; fruiting: July - November. 
Representative specimens examined: Kerala: Pathanamthitta Dist.: Sabarimala, 18.5.1997, Sivadasan CU 19152A (infl.) (CALI). Thrissur Dist.: Kuthiran, 20.5.1997, Abdul Jaleel RIA 55 (infl.) (CALI). Palakkad Dist.: Walayar, 28.7.1929, Raju \& Ratnavelu 18644 (leaf \& infr.) (MH); Walayar forest, 15.4.1979, Sivadasan CU 21442 (infl.) (CALI). Malappuram Dist.: Calicut University Campus, 26.6.1997, Abdul Jaleel RIA 96 (infl.) (CALI). Kozhikode Dist.: Mukkam, Pannicode, 30.3.1997, Abdul Jaleel RIA 65 (infl.) (CALI). Wayanad Dist.: Waithiri, 16.5.1977,

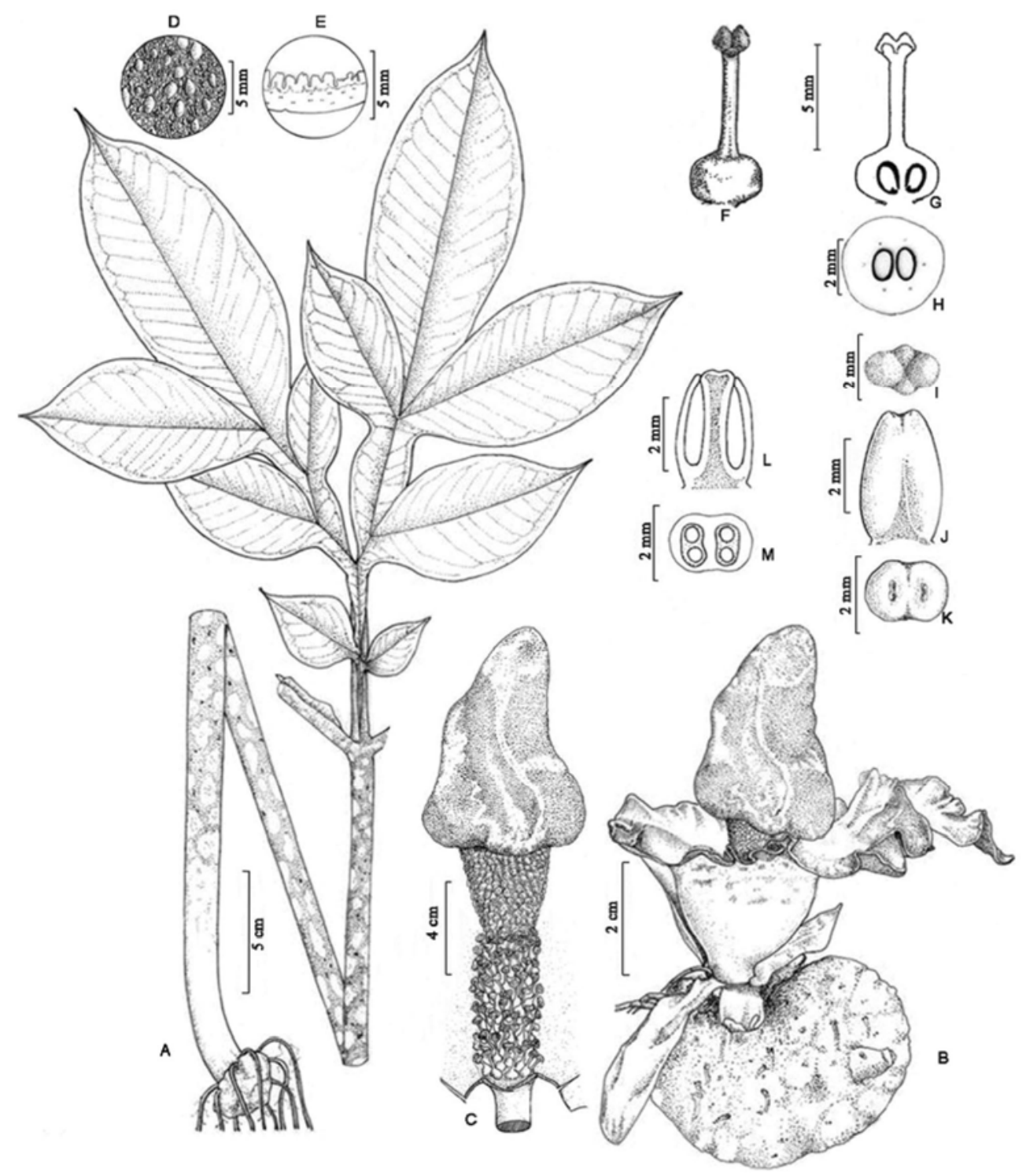

Fig. 5. Amorphophallus paeoniifolius (Dennst.) Nicolson var. paeoniifolius. A. Tuber with leaf; B. Tuber with inflorescence; C. Spadix; D. Small basal inside portion of spathe; E. A small basal portion of spathe - c.s.; F. Female flower; G. Female flower - l.s.; H. Ovary - c.s.; I. Stigma; J. Male flower - view from broad side; K. Male flower - view from top; L. Male flower - l.s.; M. Male flower - c.s. 
Sivadasan CU 19175(CALI). Kannur Dist.: Taliparamba, 16.5.1982, Nair 73916 (infl.) (MH). Karnataka: Uduppi, Padigara, 25.5.1997, Abdul Jaleel RIA 58 (infl.) (CALI). Andhra Pradesh: Sreekakulam Dist.: 17.5.1979, Subha Rao 62460 (infl.) (MH). Maharashtra: Rajpurla, 11.9.1957, Jain 24248 (leaf) (CAL). Madhya Pradesh: Indore, Mandu, 20.9.1964, Acc. No. 5980 (BSA). Orissa: Dandarkarmya, Bichacakotta coffee plantation area, 27.5.1959, Rao 18585 (infl.) (ASSAM). Bihar: Borin HB, 13.12.1957, Panigrahi 11707 (leaf) (ASSAM); Rajasthan: Rajputana, Chitargo, 29.4.1896, Prain Acc. No. 496617 (infl.) (CAL). West Bengal: Botanical Garden, Calcutta (cultivated, originally collected from Bhitorgarh, Rajputana), 3.5.1895, S. coll., s.n. (spathe) (CAL); Howrah Dist., Santra gatchi, 24.4.1896, Prain, Acc. No. 496599 (infl.) (CAL). Assam: Moshmai falls, 11.11.1938, Biswas 3928 (infr.) (CAL). Tripura: Agarthala, 10.5.1915, Debbarmar, Acc. No. 496616 (leaf) (CAL). Andaman and Nicobar Islands: South Andaman, Ograbraj, 30.11.1997, Abdul Jaleel \& Bobby Thomas RIA 219 (leaf) (CALI); North Andaman, Kalipur, 13.12.1997, Abdul Jaleel \& Bobby Thomas RIA 234 (infr.) (CALI); Nicobar islands: Car Nicobar, Lapathi - on the way to Tip Top, 12.5.1999, Abdul Jaleel RIA 339 (leaf) (CALI); Kamorta, Nancowry island, 13.5.1999, Abdul Jaleel RIA 342 (leaf) (CALI).

Note: A detailed account on identity of Rheede's (1692) Mulenschena was provided by Suresh et al. (1983) wherein nomenclatural history of Amorphophallus paeoniifolius var. paeoniifolius was elaborated.

Amorphophallus paeoniifolius (Dennst.) Nicolson var. campanulatus (Decne.) Sivad. in Suresh, Sivad. \& Manilal, Taxon 32: 130 (1983); Nicolson in Dassan. \& Fosb., Rev. Handb. Fl. Ceylon 6: 40 (1987); Nicolson, Suresh \& Manilal, An Interpr. Hort. Malab. : 274 (1988); Karth., Jain, Nayar \& Sanjappa, Fl. Ind. Enum. Monocot. : 6 (1989). [Schena Rheede, Hort. Malab. 11: 35, t. 18 (1692)]. Dracontium polyphyllum sensu Dennst., Schlüssel Hort. Malab.: 13, 38 (1818), non L. (1753). Arum campanulatum sensu auct. in part, not as to type of Roxb. (1820), nom. illegit.; Roxb., Pl. Corom. 3: t. 272 (1820); Hook., Bot. Mag. 55: t. 2812 (1828); Wight, Ic. Pl. Ind. Or. 3: 5, t. 782 (as to infl.) (1844). Amorphophallus campanulatus Decne., Nouv. Ann. Mus. Hist. Nat. Paris 3: 366 (1834); Blume, Rumphia 1: 139 (1837); Hook. f., Fl. Brit. India 6: 513 (1893); Engl., Pflanzenr. IV. 23C (48): 76 (1911). Amorphophallus dubius Blume, Rumphia 1: 142 (1837); Hook. f., Fl. Brit. India 6: 514 (1893); Engl., Pflanzenr. IV. 23C (48): 74 (1911). Amorphophallus sativus Blume, Rumphia 1: 145 (1837); Engl., Pflanzenr. IV. 23C (48): 109 (1911). Amorphophallus campanulatus var. blumei Prain, Bengal Pl.: 1109 (1903). Amorphophallus campanulatus hoofdgroep hortensis Backer, Determ.-tab. Jav. Amorphophallus: 2 (1920). Amorphophallus campanulatus f. hortensis Backer ex Backer \& Bakh., Fl. Java 3: 112 (1968).

(Fig. 6).

Type: Timor, no date, Gaudichaud s.n. (P).

Tubers depressed-globose, 8-12 cm diam. and 7-10 cm thickness in vegetative phase; 16-23 $\mathrm{cm}$ diam. and 10-12 cm thickness in reproductive phase (huge-sized tubers are also produced); skin pale brownish, with prominent root scars. Petiole 75-150 cm long, green with ovoid to elongate ovoid pale green blotches and minute pale green spots in between, extreme base white. Leaflets sessile, long leaflets $18-22 \times 5.0-6.5 \mathrm{~cm}$; small leaflets 7.5-10.5 $\times 3.0-4.5 \mathrm{~cm}$, obovate, tip acute, base unequal and decurrent on rachises, greenish above, paler below. Peduncle smooth, 6-8 $\mathrm{cm}$ long. Spathe tip acute, margin undulate, 19.0-23.5 $\times 16-19 \mathrm{~cm}$, convolute portion greenish yellow, expanded portion purplish green with white blotches; dark purplish at base within with prominent murications, middle pale green, pale purplish above, margin pale green. Spadix sessile, 20.0-21.5 cm long, female zone 4.0-4.5 cm long; male zone 5.5-6.0 cm long; appendix 8.5-9.5 cm long. Female flowers dense, each with ovary sub-spherical, pale yellowish, 2.0-2.5 mm high; 
style 7.0-7.5 mm long; stigma 3-lobed, rarely 2-lobed. Male flowers dense, each 2.5-3.5 mm high, yellowish-cream coloured. Spadix-appendix hemispherical.

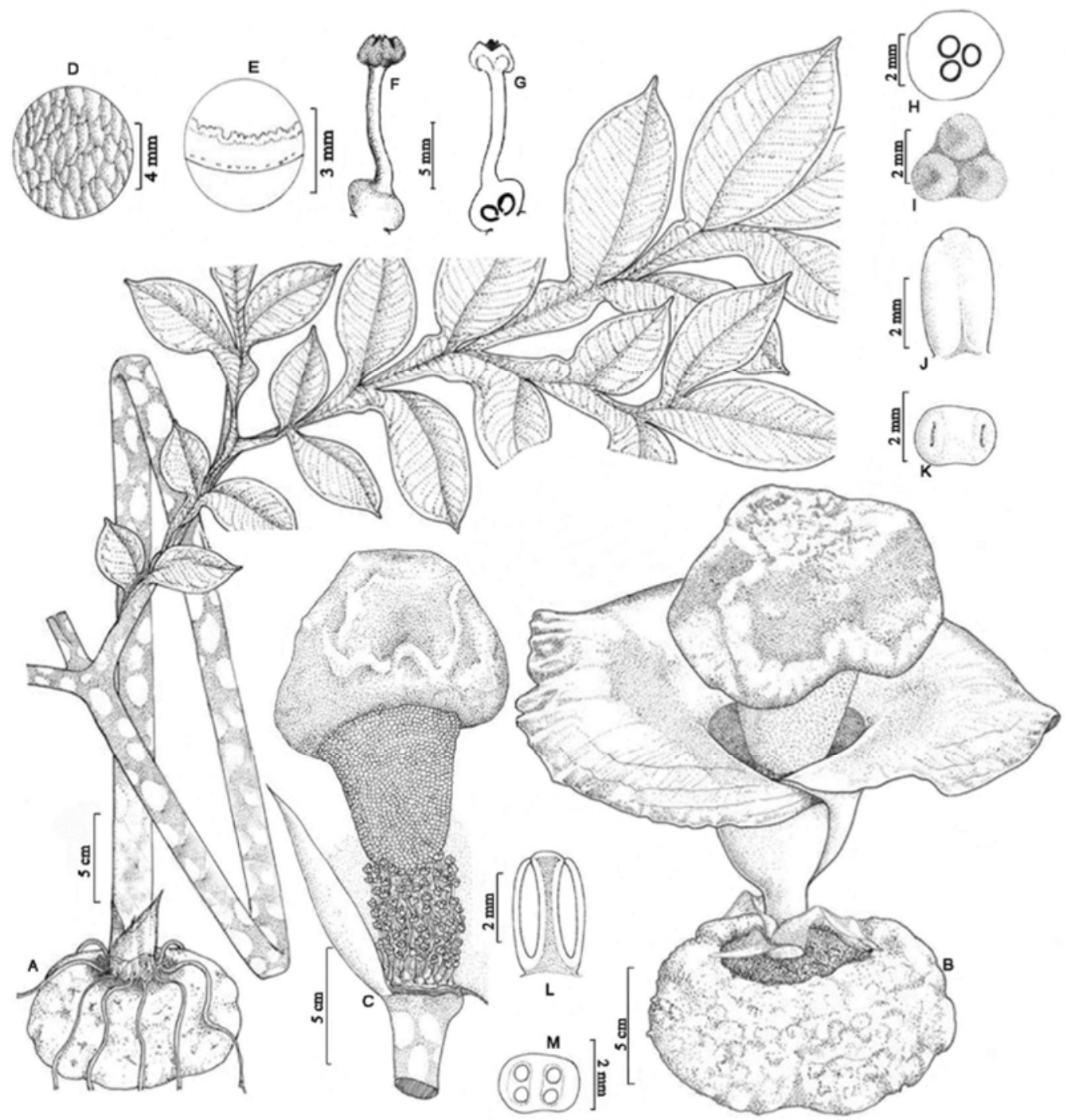

Fig. 6. Amorphophallus paeoniifolius (Dennst.) Nicolson var. campanulatus (Decne.) Sivad. A. Tuber with leaf; B. Tuber with inflorescence; C. Inflorescence - spathe partially removed showing spadix; D. Small basal inside portion of spathe; E. Small basal portion of spathe - c.s.; F. Female flower; G. Female flower - l.s.; H. Ovary - c.s.; I. Stigma; J. Male flower - view from broad side; K. Male flower - view from top; L. Male flower - l.s.; M. Male flower - c.s.

Phenology: Flowering: May - June; Fruiting: No fruit-setting.

Representative specimens examined: Kerala: Kozhikode Dist.: Areecode, 16.5.1997, Abdul Jaleel RIA 46 (infl.) (CALI); Vadakara, Azhiyur, 9.8.1997, Abdul Jaleel RIA 119 (leaf) (CALI). 
Malappuram Dist.: Calicut University Campus, 10.8.2000, Abdul Jaleel RIA 386 (leaf) (CALI). Palakkad Dist.: Athicode, Chittur, 10.4.1976, Sivadasan CU 13132 (infl.) (CALI); Kumbalakode, Elavancherry, 24.4.1997, Sivadasan CU 19170 (infl.) (CALI).

Notes: The confusion in nomenclature of the wild and cultivated varieties of Amorphophallus paeoniifolius has been sorted out by Suresh et al. (1983) while providing correct identity of aroids described by Rheede (1692).

Amorphophallus paeoniifolius var. campanulatus representing the cultivated variety differs from var. paeoniifolius in texture of petiole, characters of female flowers and spadix-appendix. The former is having smooth or slightly rough greenish petiole with white blotches, leaflet-bases not decurrent to the junction of the petiolules, style of pistil more than thrice the height of the ovary, spadix-appendix round-obtuse to broadly hemispherical with height less than its breadth, and no fruit-setting. The latter is having strongly muricate purplish petiole with light pinkish blotches, leaflet-bases strongly ducurrent usually to the junction of the three main rachises, style more or less double the height of the ovary, spadix-appendix long-conoidal, height more than its breadth, and fruit-setting.

Distribution: India, Sri Lanka, Java and Myanmar. In India, common in all States. Widely cultivated for the edible tubers.

\section{Taxonomic Analysis}

In India the genus Amorphophallus comprises three sections, viz. Amorphophallus sect. Amorphophallus, sect. Rhaphiophallus, and sect. Conophallus. Jaleel et al. (2011) stated the genus to have three sections namely A. sect. Candarum, sect. Conophallus and sect. Rhaphiophallus. But Engler's (1911) inclusion of A. campanulatus (=A. paeoniifolius), the type of the genus under sect. Candarum ("Cundarum") rendered the sectional name illegitimate. The sectional name which included the type of the genus has been correctly recognized here as A. sect. Amorphophallus.

Amorphophallus sect. Rhaphiophallus is the largest of the three sections of the genus and is represented in India by eight species (Jaleel et al., 2011), viz. A. bonaccordensis Sivad. \& N. Mohanan, A. hohenackeri (Schott) Engl. \& Gehrm., A. konkanensis Hett. et al., A. longiconnectivus Bogner, A. margaritifer (Roxb.) Kunth, A. mysorensis E. Barnes \& C. E. C. Fisch., A. smithsonianus Sivad., and A. sylvaticus (Roxb.) Kunth. Amorphophallus mysorensis is with two varieties, viz. var. mysorensis and var. bhandarensis (S. R. Yadav, Kahalkar \& Bhuskute) Sivad. \& Jaleel. Amorphophallus sect. Conophallus is the second largest of the genus in India and comprises six species, viz. A. bognerianus Sivad. \& Jaleel, A. bulbifer (Sims) Blume, $A$. carnosus Engl., A. commutatus (Schott) Engl., A. nicolsonianus Sivad. and A. oncophyllus Prain ex Hook. f. The species A. commutatus forms a complex with three varieties, viz. var. commutatus, var. anmodensis Sivad. \& Jaleel, and var. wayanadensis Sivad. \& Jaleel. The third and the smallest section is A. sect. Amorphophallus comprising five species, viz. A. hirsutus Teysm. \& Binn., A. kachinensis Engl. \& Gehrm., A. longistylus Kurz, A. napalensis (Wall.) Bogner \& Mayo and A. paeoniifolius (Dennst.) Nicolson. The species A. paeoniifolius is with two varieties, viz. A. paeoniifolius var. paeoniifolius, and var. campanulatus (Decne.) Sivad.

Among the species of A. sect. Amorphophallus, the two species, viz. A. hirsutus and A. kachinensis are new addition to the flora of India and they have been collected from Great Nicobar Island of Andaman and Nicobar Islands, and Arunachal Pradesh respectively, and their discoveries formed first reports of their distribution in India. Amorphophallus longistylus was rediscovered and collected from Andaman Islands after 131 years from the time of its first collection.

Amorphophallus paeoniifolius is the widely distributed species in India represented in all states. During the present study it was found that maximum number of species of the section occur 
in Arunachal Pradesh and Andaman and Nicobar Islands. Arunachal Pradesh has 3 species, viz. A. kachinensis, A. napalensis and A. paeoniifolius, and Andaman and Nicobar Islands have 3 species, viz. A. hirsutus, A. longistylus, and A. paeoniifolius. Amorphophallus longistylus is strictly endemic to Andaman Islands with restricted distribution and according to the criteria $\mathrm{D}$ of section V of IUCN (2012), it is considered as Critically Endangered (CR).

The fast and indiscriminate deforestation and destruction of natural habitats of the species render most of the endemic species endangered and lead to extinction. Proper monitoring of habitats and distribution are essential for conservation of species of the genus Amorphophallus which comprised wild relatives of widely used and economically important cultivated species.

\section{Acknowledgements}

The authors are thankful to Dr. Wilbert L. A. Hetterscheid, former Director of Botanical Gardens, Wageningen University, Netherlands for thorough review of a manuscript on revision of Indian Amorphophallus of which the present paper forms a part. The second author is extremely grateful towards Dr. Dan H. Nicolson, Smithsonian Institution, Washington, D.C., USA for valuable and critical discussions on nomenclatural problems during his work there. The facilities provided by authorities of various national and international herbaria mentioned under materials and methods for study of herbarium specimens are thankfully acknowledged. The authors appreciate with gratitude the valuable services rendered by Mr. V. B. Sajeev, Ernakulam, Kerala and Mr. Jayesh P. Joseph, Wayanad for the illustrations. The last four authors gratefully acknowledge the encouragements and support extended by the Deanship of Scientific Research, King Saud University, through the research group project No. RGP-VPP-135.

\section{References}

Backer, C.A. 1920. Determinatietabel voor de Javaansche Soorten van Amorphophallus Bl. N.V. Boekhandel Visser \& Co., Weltevreden, i+14 pp.

Backer, C.A. and Bakhuizen van den Brink, R.C. 1968. Amorphophallus. In: Backer, C.A. and R.C. Bakhuizen van den Brink, Flora of Java, 3. Wolters - Noordhoff N.V., The Netherlands, pp. 111-113.

Blume, C.L. 1837. Rumphia, 1. C.G. Sulpke, Leiden, Amsterdam, pp. viii+1-204.

Bogner, J., Mayo, S.J. and Sivadasan, M. 1985. New species and changing concepts in Amorphophallus. Aroideana 8(1): 14-25.

Engler, A. 1911. Araceae-Lasioideae. In: Engler, A. (Ed.), Das Pflanzenreich IV. 23C(48).Wilhelm Engelmann, Leipzig, pp. 1-130.

Hetterscheid, W.L.A. and Ittenbach, S. 1996. Everything you always wanted to know about Amorphophallus, but were afraid to stick your nose into !!!!!. Aroideana 19: 7-131.

Hooker, J.D. 1894. Amorphophallus. In: Hooker, J.D., Flora of British India, Vol. 6. L. Reeve \& Co. Ltd., London, pp. 513-519.

IUCN. 2012. IUCN Red List Categories and Criteria: Version 3.1. Second edition. IUCN, Gland, Switzerland and Cambridge, UK, pp. iv+32.

Jaleel, V.A., Sivadasan, M., Alfarhan, A.H., Thomas, J. and Alatar, A.A. 2011. Revision of Amorphophallus Blume ex Decne. sect. Rhaphiophallus (Schott) Engl. (Araceae) in India. Bangladesh J. Plant Taxon. 18(1): 1-26.

Jaleel, V.A., Sivadasan, M., Alfarhan, A.H., Thomas, J. and Alatar, A.A. 2012. Revision of Amorphophallus Blume ex Decne. sect. Conophallus (Schott) Engl. (Araceae) in India. Bangladesh J. Plant Taxon. 19(2): 135-153. 
McNeill, J., Barrie, F.R., Buck, W.R., Demoulin, V., Greuter, W., Hawksworth, D.L., Herendeen, P.S., Knapp, S., Marhold, K., Prado, J., Prud'homme van Reine, W.F., Smith, G.F., Wiersema, J.H. and Turland, N.J. 2012. International Code of Nomenclature for algae, fungi and plants (Melbourne Code) adopted by the Eighteenth International Botanical Congress Melbourne, Australia, July 2011. Regnum Vegetabile 154. Koeltz Scientific Books, Germany, pp. xxx+1-240.

Nicolson, D.H. 1977. Nomina conservanda proposita: Proposal to change the typification of 723 Amorphophallus, nom. cons. (Araceae). Taxon 26: 337-338.

Rheede, H.A. van, tot Draakestein. 1692. Hortus Indicus Malabricus, Vol. 11. Johannis van Someren et Joannis van Dyck, Amsterdam, pp. v+134 pp. +65 tabs.

Sivadasan, M. and Jaleel, V.A. 1998. Rediscovery of Amorphophallus longistylus (Araceae), a little known rare endemic species from Middle Andaman, India. Rheedea 8(1): 103-106.

Sivadasan, M. and Jaleel, V.A. 2000. Amorphophallus hirsutus Teysm. et Binn. (Araceae): A new report from India. Rheedea 10(2): 143-147.

Suresh, C.R., Sivadasan, M. and Mainilal, K.S. 1983. A commentary on Rheede's Aroids. Taxon 32(1): 126132.

(Manuscript received on 5 February 2014; revised on 4 November 2014) 